\title{
Magnification in Dentistry - Insight to the future
}

\begin{tabular}{|c|c|}
\hline $\begin{array}{c}\text { Dr. Pavithra, } \\
\text { Assistant Professor *, } \\
\text { Email : pavithraendo@gmail.com } \\
\text { Mob. } 9916353572\end{array}$ & $\begin{array}{c}\text { Dr. Roshan Uthappa } \\
\text { Endodontist } \\
\text { Private practice }\end{array}$ \\
\hline $\begin{array}{l}\text { Dr. Vinay Shivgange } \\
\text { Reader * }\end{array}$ & $\begin{array}{c}\text { * Dept. of Conservative Dentistry \& Endodontics } \\
\text { College of Dental Sciences, Davangere }\end{array}$ \\
\hline
\end{tabular}

\section{INTRODUCTION}

The great philosopher Tielhard de Chardin aptly described man as a cerebromanual creature as in the hands cannot treat what the eyes cannot see. Visual acuity is one of the pre - requisites for the successful outcome of clinical dentistry. Precise recognition of fine detail is relevant in all aspects of dentistry and is an important first step in clinical decision making. Enumerated below are the potential benefits of magnification and the different methods by which it can be obtained.

\section{HISTORY OF MAGNIFICATION}

We have come a long way since Robert Hooke discovered an image under the microscope which he termed cell. Microscopes were used by our bretheren in the medical fraternity for the last $40 \mathrm{yrs}$ in the fields of ophthalmology, neurosurgery and otolaryngology. In the year 1978, Apothekar and Jako introduced a crude form of the present day microscope and named it the dentiscope. In 1992, Dr. Gary Carr introduced an ergonomically configured operating microscope for endodontists which allowed for easy use in all dental procedures overcoming the disadvantages of the dentiscope. ${ }^{1}$

\section{WORKING OPTICAL PRINCIPLES}

Resolution is defined as the ability to distinguish separately two objects that are closely placed. The resolving power of the unaided human eye is $0.2 \mathrm{~mm}$. Dentists often must distinguish microscopic details that are beyond the resolution of the human eye. Minute details such as early carious lesions, cavosurface margin defects, crown margin evaluation and microcracks are all diagnostically difficult to do without an improvement in our native visual acuity. With magnification, the resolving power of theeye can be improved to $0.006 \mathrm{~mm}^{2}$

\section{VARIOUS MAGNIFICATION DEVICES}

Of all the magnification systems available today, loupes have been quite popular. Loupes are essentially two mono ocular lenses mounted side by side and angled inward to focus on an object. Loupes are economical, easy to get started with and their curve is much shorter than for the microscope. However, there are several other differences that need to be explained.

Loupes have a convergent lens system, the user's eyes must converge to view a sharp image. This can cause eyestrain, fatigue and vision changes if not adjusted properly. They have a fixed magnification of upto $4 \mathrm{X}$ that cannot be changed. Any further increase in the magnification leads to bulkier loupes which are not user friendly.

The focus in loupes is made by either moving the patient or the operator's neck up/down hence compromising the ergonomics. The operating microscope on the other hand has a shadow free co - axial illumination with a wide range of magnification. Also documentation is possible with good ergonomics. $^{2}$

\section{APPLICATIONS OF THE MICROSCOPE}

New perspectives for endodontics : In endodontics, magnification of upto $25 \mathrm{X}$ makes it possible for us to recognize all root canals, isthmuses,perforations,microfractures,leakages in existing fillings and visualize down to the apex. ${ }^{3}$

Restorative Dentistry: there is unparalled precision, selective diagnosis of caries, extremely precise recognition of enamel and dentin fractures and predictable treatment outcomes for maximum patient satisfaction. $^{4}$

Prosthodontics : while making a wax pattern for indirect restoration, the microscope is an ideal tool for marginal contouring. Overhangs can be eaisily appreciated and removed. ${ }^{2}$

Oral Surgery: The surgical operating microscope can be highly beneficial for procedures like microvascular reconstructive surgeries and temperomandibular joint reconstruction. ${ }^{2}$

Implant Dentistry: The placement of dental implants demand clinical performance that challenge the technical skill of dentists beyond the range of ordinary visual acuity. ${ }^{2}$

Periodontics: The use of microinstruments and 
extremely fine suture material results in precise instrument guidance and hence faster healing, reduced scar formation and improved cosmetic outcome. $^{5}$

\section{Figure 1: Conventional Vs Microsurgery}

Pedodontics:Children are fascinated by the possibility of observing their own treatment, watching the reflection in the lens. Observing occlusal fissures through the microscope is particularly impressive as they are inherently fine and difficult to see.

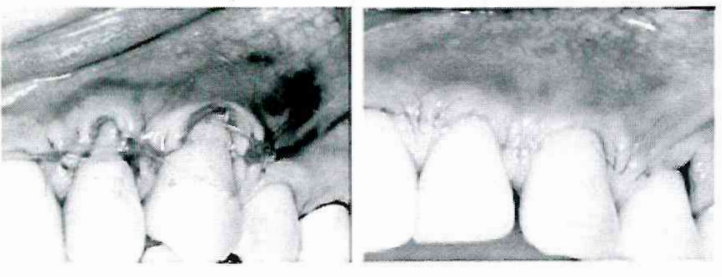

Beyond the endodontic practice, the role of the microscope has received increased attention in general dentistry to further enhance the quality of the dentists' work, obtain esthetic outcomes and conserve long term dental health for patients. ${ }^{2}$

\section{Frequently asked Questions about the Dental} Microscope ( FAQ's )

1. What is the added value of a microscope for dentistry in general ?

Dental procedures can be performed more accurately and reliably with varying magnification and excellent illumination. Recorded images can be used for patient counselling and it has rightly been said that an image says more than a thousand words.

2. What is the added value of a microscope for endodontics?

It is easier to locate difficult to access canals and to visualize isthmuses, perforations, split or oval canals. Defects in hand and rotary files can be traced at an early stage resulting in the likely prevention of instrument separation.

3. Does one have to get used to operating a dental microscope?

Working under magnification requires a training periodand time for the operator's hand - eye co ordination to develop. At first, people are mainly surprised about the level of detail and things that previously remained unseen. Treatment might be slightly slower in the beginning. Eventually, procedures can performed more predictably and faster with a dental microscope.

4. Are special instruments required for working with a dental microscope?

Working with a dental microscope requires microin- struments designed to work in the microenvironment in focus. Drills with longer shanks are preferred for better visualization.

5. What accessories does a dental microscope include?

Apart from video recording and capturing still images, a co-observation tube attached to the microscope housing allows the assistant to follow the procedures as they are being done..$^{2,6,7}$

\section{OTHER MAGNIFICATION DEVICES}

Figure 2: Endoscope

\section{Endoscope}

Limited intracanal visualization in endodontics was the catalyst for fiber optic development and usage. In addition to magnification and illumination afforded by the microscope, endoscopy offers the advan-

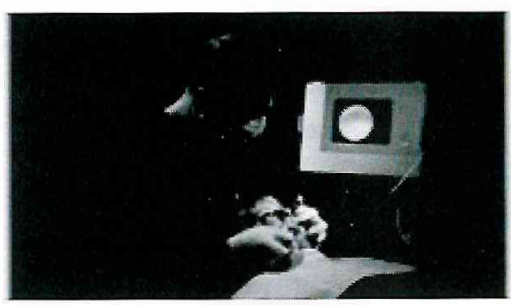

tage of "circumferential"vision of the entire region making it possible to examine the lingual surface of the root or the vestibular wall of the prepared retro cavity. ${ }^{8}$

Orascope

\section{Figure 3: Orascope}

It is a modified medical endoscope designed for visualisation in the oral cavity. The actual

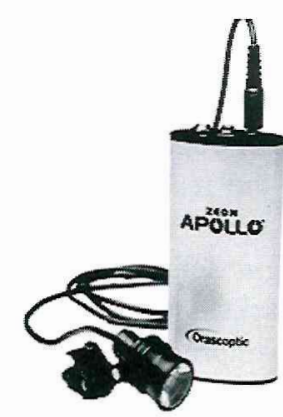

orascope/endoscope usage during conventional or surgical treatment is minimal. It is used when specific magnification is needed for critical evaluation. It could be used for intra - canal visualization and to view a treatment field from various vantage points without losing depth and focus. The operator holds the orascope instead of the dental mirror whilst the 
assistant retracts the surrounding tissues and maintains hemostasis. ${ }^{8,9}$

\section{CONCLUSION}

Magnification not only offers many useful applications in the treatment of root canals and throughout the entire field of dentistry, but also improves the overall treatment quality. It encourages dentists to review and perfect their own treatment concepts resulting in a positive impact on the entire practice structure.

\section{REFERENCES}

1. Apothekar H Jako GJ. A microscope for use in dentistry. J microsurg 1981;3(1): 7-10

2. Enrique Merino. Textbook of Endodontic Microsurgery

3. Suehara $M$, Nakagawa $K$, Aida N, Ushikubo T, Morinaga K. Digital video image processing from dental operating microscope in endodontic
treatment..Bull Tokyo Dent Coll. 2012;53(1):27-31.

4. Sheets C G, Paquette J M. Microscopy in dentistry. Esthetrestor dent 2003;15(7):390-410

5. Shanelec D A,Tibettss L S. Periodontal microsurgery, periodontal insights 1994;1(5):4-7

6. Mounce $R$. The surgical operatingmicroscope: pushing the boundaries of the possible in dentistry. Dent Today. 2006 Oct;25(10):108, 110, 112-5.

7. Syngcuk Kim. Color Atlas of Microsurgery in Endodontics

8. Moshonov J, Nahlieli O. Endoscopy in endodontics. Alpha Omegan. 2011 Spring;104(1-2):26-34.

9. Keiser K.Magnification options in endodontics: A comparison of the operating microscope and the endoscope.Tex Dent J. 2007 Feb;124(2):208-16 